\title{
New Cloud Economy For Resolving A Problem Cloud Computing Adoption
}

\author{
M. Elmahouti, O. Achandair, S. Khoulji, M. L. Kerkeb, \\ Information Systems Engineering Research Group, Dept. Telecommunications. \\ National School of Applied Science of Tetouan, \\ Abdelmalek Essaadi University, Tetuan, Morocco \\ mouradelmahouti@gmail.com, o.achandair@gmail.com,khouljisamira@gmail.com, \\ kerkebml@gmail.com,
}

\begin{abstract}
Cloud computing, IT still called to order, it is a model that allows access to information resources on demand, we can consider it as a unique Technology to satisfy needs and respond to customer demands by guaranteeing an efficient quality of servicelT management.

This paper gives an analysis of cloud computing along its components, also the internal and external institutional factors that influence the adoption of cloud computing in the majority of enterprises.

It aims to be innovative by answering these questions of allocation and availability of network services in an intra-datacenter environment by implementing solutions based on new technologies that is OpenStack[1].

The research problem addressed in this paper is the proposal of a new architecture and optimized algorithms of continuity and availability of the service. These will help identify the deployment of existing vulnerabilities. On the other hand, offer a very high level of availability, security and quality of service.

Keywords-Cloud computing, Private cloud, Public cloud Information resources, Information security, OpenStack

\section{Introduction}

Today cloud computing is one of the information technologies that shows an intrinsic transformation for improving the quality of service rendered. The goal is to minimize the reflection time on the processing of an exponential amount of dynamic data. Today, it is difficult to cope with the increasing difficulties for a good functioning of the different architectures, the new concept cloud computing provides a fascinating proposition to the companies to outsource the IT infrastructures.

Generally, four resources can be used through the Internet and by providing an extended economy, starting with the infrastructure system like storage, the second type is the software system that supports the middleware part or other parts, and the application system as is the case of GOOGLE, finally the commercial procedures.
\end{abstract}


M. Elmahouti, O. Achandair, S. Khoulji, M. L. Kerkeb. New Cloud Economy For Resolving A Problem Cloud Computing Adoption. Transactions on Machine Learning and Artificial Intelligence, Vol 5 No 4 August (2017); p: 411-425

This article describes the design of cloud computing, the design of the architecture adopted, the points gained from this model and an analysis of the major data technologies, as well as an understanding of the factors that hamper the adoption of this model.

The article also responds to the concerns expressed today by most information systems managers who have not been able to advance in the adoption of this new cloud computing technology because they have recently invested very large budgets for their current platforms, presenting them with an intelligent solution that meets future needs with a saving of budgets reserved for the IT part, the solution is based on the free software OpenStack[3]., which consists in setting up a flexible cloud "Private- Public". A private cloud that supports their current platforms and a public cloud that manages their loads when exceeding the company's internal technical capabilities by ensuring a high level of data exchange security between the two clouds.

The article gives a presentation on the architecture, synopsis and types of cloud computing, discussing the benefits of adopting this technology, and the internal and external factors that hampered the rapid adoption of the cloud by most Presenting an effective technical solution to meet the constraints encountered.

\section{Cloud computing : Synopsis}

Cloud computing is a computer system based on the use of the Internet to exploit IT resources[2]., these resources are used through a network approach, connecting to a pool that shares configurable resources such as servers, storage, networks And applications that can be directly assigned to customers by service providers.

Cloud computing consists of some basic aspects:

- $\quad$ Measured service: The IT resources are shared by various customers, ensuring a measure of usage of each, with control and monitoring, which gives clarity to both parties: consumer and supplier.

- $\quad$ Resource pooling: The provider of cloud computing makes available to its various clients resources in common using the multi - support mode to share the various virtual and physical resources with a possibility of renewal according to the demand of the client[4].

- Rapid elasticity: Great flexibility in the release and allocation of resources, real-time adjustment, with a perfect control of the consumption of resources.

- On-demandself-service: The existence of an application that responds spontaneously to allocate resources automatically without going through a human intervention.

\subsection{Architectureof Cloud Computing}

The architecture of cloud computing[5]. can be divided into four layers, starting with the application layer, the layer that forms the platform, the infrastructure layer of the party and the hardware layer as shown in "Figure 1".

- Application layer : This is the top level of cloud model composed specific cloud applications, while different from conventional applications, it will secure the automatic scale part to get great performance with reduced costs.

- $\quad$ Platform layer: It is the secondary layer that forms the operating structure, whose role is to reduce 
the load by extending the application directly to the storage VM.

- Virtualization layer: Called the infrastructure layer that remains fundamental to the cloud system, which ensures a dynamic aspect of the various components based on KVM virtualization technologies such as the storage pool

- Hardware layer: This is the bottom layer of the architecture of the cloud, responsible for maintaining the physical cloud resources, such as routers, cooling systems and power, this layer contains a number of interconnected servers switches and other devices.

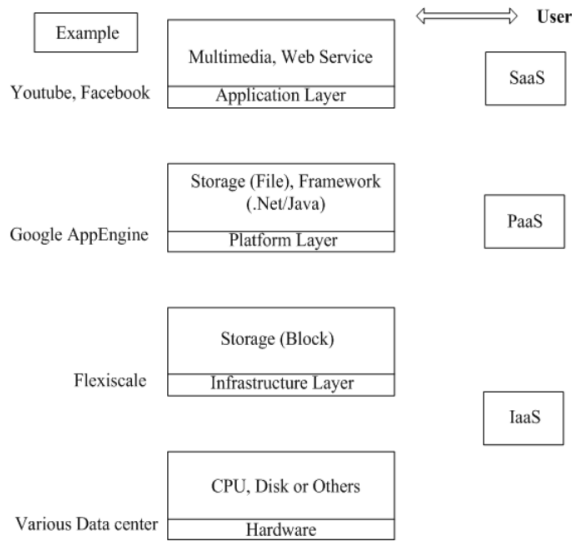

Figure1: Architectural View of Cloud Computing

\subsection{Cloud Hierarchy}

In most scenarios, the Cloud hierarchy is provided by four levels of users, first level users, field staff who are the first users who coordinate basic services, then transaction authors, and finally developers $(\mathrm{Cl})$, See "Figure 2".

- User:These are the end users, in this layer we even find indirect users like decision-makers on policies to choose, analysis and illustration.

- Service integration and composition: At this level, the user judged expert must be able to focus on training results intended for an end user can integrate and manage existing services, able to customize and provide updated services and inspect the use of services and collect service information.

- Service author: the authors are the developers of distinct bases of services that can be used directly or combined in other aggregates, and complex service systems by experts in providing the service. The authors should be experts components having a positive outlook needs and categories to ensure such registration services, license management tools, fault tolerance.

- Developers: Cyber-infrastructure (CI) developers are responsible for developing cloud structure, be experts is an asset in the areas of storage, middleware, networks, generation systems, creating devices for services, scheduling algorithms and others[6]. 


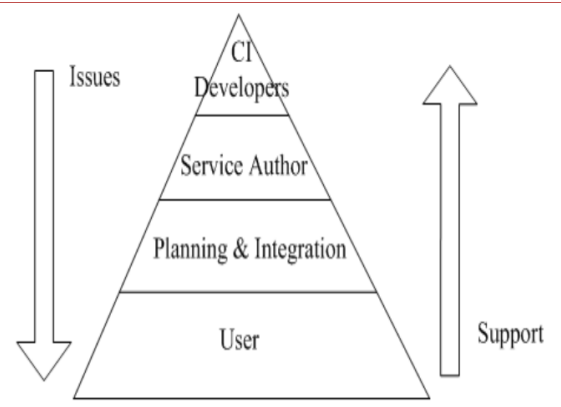

Figure 2: The Hierarchy of Cloud Computing

\subsection{Service Model of Cloud Computing}

Cloud management is completely centralized. The database server has the role to specify the balance between customer demand and system capabilities, and monitor network traffic.

Users achieve IT infrastructures from clouds system and next run applications inside thus Clouds computing distribute customer along services to approach software, infrastructure and data resources as shown in "Figure 3".

- Infrastructure as a Service: laaS Infrastructure as a Service is one of three main categories of cloud computing services, this service gives access to computing resources in a virtualized environment, the "Cloud", through a public connection, typically the Internet. For laaS, the resource is supplied hardware virtualized or, in other words, an IT infrastructure. The service definition includes offerings such as server space, network connections, bandwidth , IP addresses and load balancers . Physically, the hardware resources come from a multitude of servers and networks typically distributed across many data centers, the cloud provider's responsibility to maintain. Meanwhile, access to virtualized components is given to the client company so that it can build its own IT platforms. [7].

- Platform as a Service: often simply referred to as PaaS, is a category of cloud computing that provides a platform and environment to allow developers to build applications and services over the internet. PaaS services are hosted in the cloud and accessed by users simply via their web browser.

- Software as a Service: Any cloud service that allows customers to have access to software applications via the Internet or Internet as if applications hosted in a service provider. These applications are hosted in the cloud and can be used if they have permissions, both by individuals and by organizations. Google, Twitter, and Facebook are all examples of SaaS, or users can connect to the services by any device with an Internet connection. 


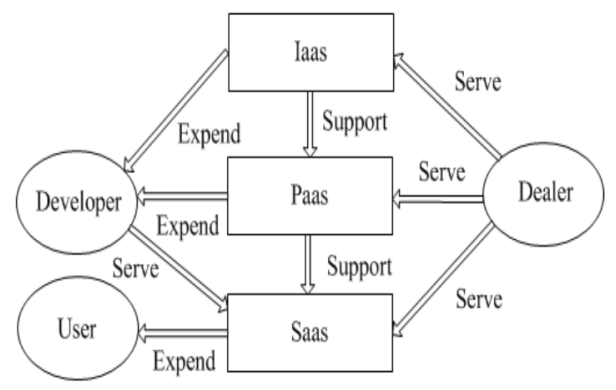

Figure 3: Abstractions of Cloud Computing

\section{Types of cloud}

There are different types of cloud that allow a wide choice for users in the desired formula, namely the private cloud, public cloud, hybrid cloud and virtual private cloud.

- Private cloud: this model is mainly chosen to provide the client more control and privacy on his cloud based environment, which consist of offering a pool of physical computing resources as a service accessible through a virtualized environment.

- Public cloud: This type of cloud is to make available a shared platform to serve an unlimited number of users via the Internet. Also, this means optimal use of virtualized physical resources, and therefore a consumption model based on 'you pay as yougo'. The entirely infrastructure that host client environment belong to the cloud provider, who is responsible of all the management and maintenance layers[8].

- Hybrid cloud: it is about coexisting and communicating a private cloud and a public cloud. This type of cloud is often used for the purpose of rise-time load as permitted by public cloud. The only difference is that in this case it would be linked to a private or internal cloud for communicating the two infrastructures.

Virtual private cloud: This model is a hybrid cloud environment provided by the public cloud; the cloud provider guarantees an access to an isolated portion of his infrastructure for private use. Virtual private cloud is offered through a secure connection between the enterprise and the provider. The entire infrastructure belong the provider who is responsible for all the public cloud operations in addition to isolating the concerned portion[9].

Table I: Comparison of various representative cloud platforms

\begin{tabular}{|c|c|c|c|}
\hline \multirow{2}{*}{ Feature } & \multicolumn{3}{|c|}{ Organization } \\
\cline { 2 - 4 } & Google & Amazon & Microsoft \\
\hline Focus & Platform & Infrastructure & Infrastructure \\
\hline Business Type & $\begin{array}{c}\text { Web } \\
\text { Application }\end{array}$ & $\begin{array}{c}\text { Storage, } \\
\text { Compute }\end{array}$ & Storage \\
\hline Virtualisation & $\begin{array}{c}\text { Application } \\
\text { container }\end{array}$ & $\begin{array}{c}\text { Xen } \\
\text { hypervisor }\end{array}$ & OS level \\
\hline Web APIs & Yes & Yes & Obscure \\
\hline $\begin{array}{c}\text { Compute } \\
\text { Scheme }\end{array}$ & Python & Linux AMl & $\begin{array}{c}\text { Not } \\
\text { applicable }\end{array}$ \\
\hline
\end{tabular}


M. Elmahouti, O. Achandair, S. Khoulji, M. L. Kerkeb. New Cloud Economy For Resolving A Problem Cloud Computing Adoption. Transactions on Machine Learning and Artificial Intelligence, Vol 5 No 4 August (2017); p: $411-425$

\section{The benefits to adopt cloud computing for other data analysis technologies. (Example: BigData)}

Big data background:

The exponential growth of data, generated continuously in different formats and from different sources, has lead traditional architecture and infrastructures to face many limitations to store and process such data sets, organizations are getting more and more convinced that the cloud computing is the right structure to handle their big data projects. Big data technologies start when traditional database management and traditional processing applications are facing limits and difficulties to perform[10].

In the following, we are going to describe important big data characteristics and cloud major benefit for big data.

Gartner and many IT organizations used the so called ' $3 V^{\prime}$ ' model to describe big data, which stands for :

- Volume: huge amount of data are generated from different sources such as social media, Internet of Things (IoT), mails, purchase transaction records and many others. It is estimated that $90 \%$ of the existing data has been generated during the last two years according to a new infographic compiled by Singapore- and India-based Aureus Analytics. Storing such data sets is actually challenging the researches, it need a highly scalable and reliable storage withminimal costs. Traditional systems are still needed like SAN (storage area network), still too expensive, but not designed to handle this volume/types of data, also the remaining time to query the data from it could presents many issues while performing complex computing. Most of the big IT players rely on Hadoop for storing the data in a distributed file system mechanism, which allow the storage to scale up to massive amount [11]..

- Velocity: Data is generated at increasing speeds, accessing all of it is not usually enough. People need to access the data at the right time [12].. While Hadoop map/reduce is more suited for batch-processing data, the high velocity data is processed with other real time analytics engine that complete the Hadoop ecosystem, essentially:

Table II: Comparison of various representative cloud platforms

\begin{tabular}{|c|c|c|}
\hline package & Use case & Used by companies \\
\hline Storm & $\begin{array}{l}\text { high-speed, event processing } \\
\text { system that allows for } \\
\text { incremental computations }\end{array}$ & Twitter, \\
\hline Spark & $\begin{array}{l}\text { must have stateful } \\
\text { computations and exactly- } \\
\text { once delivery; doesn't mind } \\
\text { higher latency }\end{array}$ & $\begin{array}{l}\text { Yahoo!, NASA JPL, } \\
\text { eBay }\end{array}$ \\
\hline Samza & $\begin{array}{l}\text { has a large amount of state to } \\
\text { work with }\end{array}$ & $\begin{array}{l}\text { Linkedln, Yahoo!, } \\
\text { Metamarkets }\end{array}$ \\
\hline
\end{tabular}

- Variety: it is about collecting data in different formats from different sources, structured and unstructured, good and bad mixed together. in our networked world, systems are connected 
every day in different level of dependencies. enterprises needs to track a large amount of systems that are getting upgraded or changed continuously, managing and modeling thousands of data-type to be filtered and analyzed is a big challenge for industry of big data especially when its coupled with the previous problems of volume and velocity[13].

- Cloud Convenience for big data: The Cloud computing offer many benefits for big data industry, principally as an infrastructure that support large-scale physical resources, networking, computing, high capacity of storage, high availability, high reliability... In parallel of that, its model is too attractive because it offers companies the possibility to reduce business costs, related to the investment and maintenance of their IT infrastructure. The maturity of the public cloud model driven by big IT players as Google, Amazon and Rackspace have pushed many enterprises to migrate toward cloud platforms[14].

\section{External and Internal Institutional Factors that Influence the Adoption of Cloud Computing}

This section describes the main factors that have influenced the speed of adoption of cloud computing technology by a large majority of companies, according to a survey of 100 heads of information systems in Morocco. Are classified into two families, external and internal factors:

\subsection{External Factors:}

The external factors that inhibit the adoption of this new mode of service are the following:

- Absence of regulatory organizations

- Service providers

- The media influence

- Managing change and corporate culture.

- The lack of clear legislative law in the event of breach or termination of contract between the two parties concerned: customers and service providers.

- The lack of security especially on the confidentiality of the data is the first brake enunciated by the potential DSI.

- A market that demands to be monitored to stop falling into the serious drifts that may be the resale of personal information after being pirated.

- The complexity and lack of integration for cloud adoption by companies, according to a new panEMEA study by Oracle. Many companies have a bad approach to cloud computing in computing and business.

- Animportant aspect of the problem is that most companies continue to finance their IT investments without aligning them with potential revenues and innovative projects: two out of three decision-makers say that IT funding is too traditional and hinders innovation: It's time to change funding models.

- Companies with an established position, such as major software companies, infrastructure providers, companies that provide services, etc. Cloud computing challenges their business model. They know they have to do it, however, much of their revenue is not realized by the cloud.

- Internet networks play a very important role in the adoption of "cloud computing" technology, telephone operators must improve the current quality of the supplied data that does not respond properly to the needs desired by the companies. 
M. Elmahouti, O. Achandair, S. Khoulji, M. L. Kerkeb. New Cloud Economy For Resolving A Problem Cloud Computing Adoption. Transactions on Machine Learning and Artificial Intelligence, Vol 5 No 4 August (2017); p: 411-425

- Lack of efficient data centers.

\subsection{Internal Factors}

According to an in-house research of companies, exactly in the IT departments, there are several concerns that slow down the speed of adoption of the new technology of Cloud Computing, to quote exactly:

- According to a local study, the age pyramid of IT decision-makers in Morocco is between 48 and 55 years, which makes it difficult to adopt, and in particular decision-making migration to thistechnology, which requires know-how and a large capacity To ensure control while there is a great lack of knowledge of this type of services.

- Concerns about reducing the number of positions in the IT departments and the loss of current positions of responsibility following the organizational changes that will accompany the adoption of Cloud Computing.

- The immediate ICT environment within their company blocks progress.

- Current equipment has cost too much to be replaced so quickly

- The complexity of network architectures already present in companies

- There is also mistrust on the part of IT managers who are afraid of losing power or even seeing their leadership disappears.

- IT managers believe that the adoption of the cloud will slow down the career development of their employees, IT managers will only have to interface the internal demands of the business with Cloud service providers.

\section{Private-Public Cloud Model based on OpenStack}

To encourage companies to adopt this new cloud computing technology, minimizing budgets in the future and taking advantage of the state of the existing and existing platforms, our proposal is to create two models "Private : Cloud A"And" public : Cloud B "cloud based on a free solution that is the OpenStack[15].

Our approach is to create a "private - public" cloud: a private cloud (Cloud A) that supports the existing platform, and once the number of requests exceeds the capacity of the private platform, these requests will be refused internally by routing them to the Cloud to satisfy them by the public cloud (Cloud B) by paying just the rental costs, And once the internal resources are released, automatically some of the applications running on the public cloud will be supported again by the private cloud by minimizing rental costs[16].

\subsection{Architecture proposed for the cloud "Private - Public"}

The "Figure 4" shows the mechanism to be used by proposing the three algorithms to follow in order to pass the two-way passage between the two clouds, private and public, to ensure this proposition: 


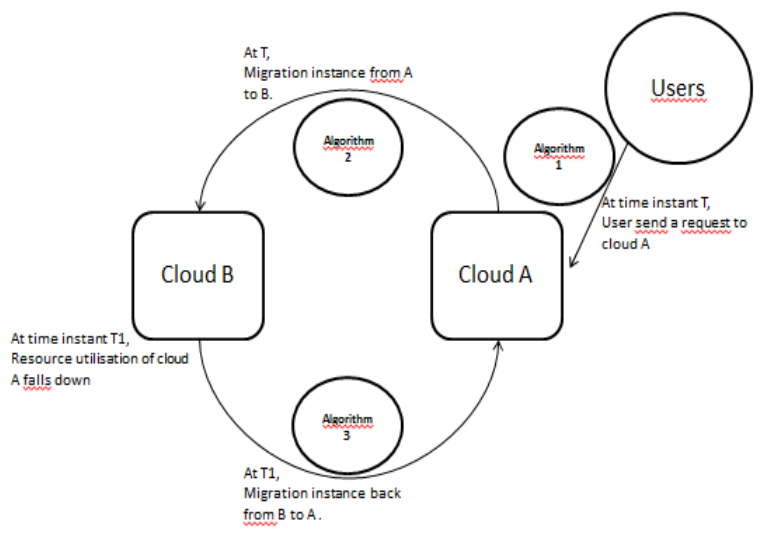

Figure4: Cloud integration

\subsection{Presentation of the three algorithms used:}

The main brick of Openstack is Nova. Its purpose is to manage the resources of calculation of the infrastructure used, using the command NOVA BOOT to attack our platform, with its syntax below:

nova boot --image imageID --flavor flavorID --nic net-id=nicID

imageID :To select the operating system.

net-id=nicID : To tell the cloud which subnet is used for an instance.

flavorID :Reflects memory, disk and virtual process requirements.

To check the actual load on the cloud A, an implementation of the load analysis function is done, the functioning of the "analyze load" function will be explained in the algorithm 1 below[16].

Once the "analyze load" function is called, all information from all compute nodes of a controller on cloud $A$ is received, if the load on the A cloud is below the configurable threshold, the instance will be created On cloud A, but if the load is greater than the threshold, the instance will be created on the cloud $B[17]$. 
Algorithm 1 Analyzing the load

Input Parameter:

flavorid: flavor id of requested instance

Output Parameter:

val: 0 if cloud is overloaded, 1 otherwise.

procedureAnalyzing the load ( $f$ lavor id)

Oracle database connection is made to the nova

database.

Extract user's requirements using flavor id from the instance types table.

totalmemory for all compute and total disk for all compute is initialize $\bar{d}$ to 0 .

freememory for all compute and free disk for all compute is initialized to 0 .

for each compute node $c$ do

Find the total memory and the free memory of $c$

from the compute nodes table.

Find the total disk space and the free disk space of c from the compute nodes table.

totalmemory for all compute =total memory for all compute + total memofy of $c$.

total disk for all compute $=$ total disk for all c-

ompute + total disk of $\bar{c}$.

freememory for all compute $=\overline{\text { free }}$ memory for all c-

ompute + free memory of $\bar{c}$.

free disk for all compute $=\overline{f r e e} \overline{\text { disk }} \overline{-}$ for all co-

mpute + free $\overline{\text { disk of }} \mathrm{c}$.

end for

if (total disk for all compute * disk threshold

percentage $>=$ users disk requirement + (to-

tal disk for all compute-free disk for all 
compute) then $\quad-\quad-\quad$

if (total memory for all compute* ram threshold

percentage) $>=$ users memory requirement +

(total memory for all compute-free memory for

all compute) then

Return 1

end if

end if

Return 0

end procedure

Note that setting up an instance on another cloud requires some information on the Cloud B: Image-ID, Network-ID, which is used by algorithm 2 to position the instance on the cloud B[18].

Algorithm 2 Position the instance on the cloud B

Input parameter:

Network id: Subnet to start instance. Image id: OS launching an instance.

Tasting Id: Specify required resources

Instance Name: The name of the instance to be launched.

Procedure INSTANCE positioning ()

When running the nova boot command, the function creation ()

calls up the load to check the load

If Not exceeding threshold then

The instance is created on cloud A.

Other

It calls the createlnstance () function. // Creating the instance on the cloud B

Createlnstance () uses the new startup parameters

We call the function creation () and extract the

Id of the image for the cloud B.

It Execute the NOVA command with the new parameters.

This nova boot command is executed

On the B cloud using the SSH connection. 
M. Elmahouti, O. Achandair, S. Khoulji, M. L. Kerkeb. New Cloud Economy For Resolving A Problem Cloud

Computing Adoption. Transactions on Machine Learning and Artificial Intelligence, Vol 5 No 4 August (2017);

p: 411-425

All instance information is stored in a file, retrieved later, and stored again in the array of migrating instances to the NOVA database

End if

End of procedure.

After each migration to the cloud $B$, the load on the cloud $A$ is verified, once the load is below the configurable threshold, a remigration is made again to the cloud $A$, the instances that will be migrated back to the cloud A follow A FIFO order, algorithm 3 below explains the function of the remigration operation:

Algorithm 3 Remigration

Input Parameter:

Migratedinstancestable: Table regrouping the instances of remigration

procedure REMIGRATION( )

The Analyzing the load() for remigration is Performed at

Of the parameterized intervals.

if the resource utilization falls below a configurable

low threshold then

do

It finds the instance to be remigrated from the

migratedinstances table using the FIFO : rule.

It launches a new instance on cloud A using

the information stored in the migrated instances table.

It copies the disk image of the migrated

instance from the cloud B to the newly launched instance on the cloud $A$, to: restore the current state.

It finished the remigrated instance from thecloud $B$ to free resources.

It cleans the entry of the table of instances that have been migrated.

whileResource usage is below the migration threshold and the migrated instance table is not empty

end if

end procedure 
The communication between the two clouds (Cloud A and Cloud B) is secured with the use of an SSH public key, as noted in algorithm 2.

\section{Results}

Currently, OpenStack installations have been deployed in the VEOLIA data center (Pilot Site: Gabon) on two dell poweredge 2950 servers. The first server acts as a private cloud (Cloud A) and the second as a public cloud (Cloud B). The memory of each server is: $1.8 \mathrm{~TB}$, which acts as one of the compute nodes. 30 machines were assigned which function as calculation nodes for the two clouds, respectively. Knowing that the two clouds belong to a single subnet[19].

The three proposed algorithms are already tested, we manage to control the load on the private cloud inhouse (Cloud A), once the configurable threshold is exceeded, the new instances launched at the company's breasts are migrated and directly supported To the public cloud (Cloud B), with a check every 30 seconds of the capabilities of the cloud $A$, once the load falls below our internally configurable threshold, the instances launched on the cloud B are again remigrated and retrieved by the cloud $A$ by following a FIFO (First In First Out) recovery order, which will also minimize our rental costs at the public cloud provider[20].

The next step will be to generalize the study across our platform by creating a private cloud that contains the entire existing infrastructure, with the proposal of a powerful test server that will play the role of the public cloud, Sharing of all the screenshots of both implementations will be ensured to facilitate the task for our service provider for a rapid adoption and implementation of this new approach by defining the contracts to respect for both parties: VEOLIA and his service provider.

\section{Conclusion}

In this paper, we proposed an efficient solution for extending the technical capabilities and performance of a platform, based on the OpenStack free solution for the creation of two flexible clouds. The first private cloud contains our current platforms and the second cloud Hosted by our service provider will serve as a platform for reinforcement when necessary in the event of internal overhead, this approach will allow us to minimize future costs and budgets, which will be limited to a resource allocation according to the timing of consumption in place Purchase and day-to-day management.

The idea is to apply this approach to all of the company's subsidiaries worldwide, a private cloud for each subsidiary and then consolidate them into a single global private cloud that will be supported by our service provider's public cloud When the configurable thresholds are exceeded.

\section{ACKNOWLEDGMENT}

This work was carried out with the support of Our home affiliation, for the implementation of the "VALVEOL - Cloud" project.

We thank all members of the project team who contributed to the success of this proposal. 
M. Elmahouti, O. Achandair, S. Khoulji, M. L. Kerkeb. New Cloud Economy For Resolving A Problem Cloud Computing Adoption. Transactions on Machine Learning and Artificial Intelligence, Vol 5 No 4 August (2017);

\section{REFERENCES}

[1] Haifeng Li, Huachun Zhou, Hongke Zhang, Bohao Feng, "EmuStack: An OpenStack-Based DTN Network Emulation Platform", 2016 International Conference on Networking and Network Applications (NaNA), vol. 00, no. , pp. 387-392, 2016, doi:10.1109/NaNA.2016.24

[2] Dinkar Sitaram, Sudheendra Harwalkar, K.V. Suchith Kumar, "Standards Based Integration of Intercloud for Federation with OpenStack", 2016 IEEE International Conference on Cloud Computing in Emerging Markets (CCEM), vol. 00, no. , pp. 113-118, 2016, doi:10.1109/CCEM.2016.028

[3] Pooya Musavi, Bram Adams, Foutse Khomh, "Experience Report: An Empirical Study of API Failures in OpenStack Cloud Environments", 2016 IEEE 27th International Symposium on Software Reliability Engineering (ISSRE), vol. 00, no. , pp. 424-434, 2016, doi:10.1109/ISSRE.2016.42

[4] M. Abhishek Sharma, Monica O. Joshi, "Openstack Ceilometer Data Analytics \& Predictions", 2016 IEEE International Conference on Cloud Computing in Emerging Markets (CCEM), vol. 00, no. , pp. 182-183, 2016, doi:10.1109/CCEM.2016.045

[5] Ali Babar, Ben Ramsey, "Tutorial: Building Secure and Scalable Private Cloud Infrastructure with Open Stack", 2015 IEEE 19th International Enterprise Distributed Object Computing Workshop (EDOCW), vol. 00, no. , pp. 166, 2015, doi:10.1109/EDOCW.2015.23

[6] Dario Bruneo, Francesco Longo, Giovanni Merlino, Nicola Peditto, Carmelo Romeo, Fabio Verboso, Antonio Puliafito, "A Modular Approach to Collaborative Development in an OpenStack Testbed", 2015 IEEE Fourth Symposium on Network Cloud Computing and Applications, vol. 00, no. , pp. 7-14, 2015, doi:10.1109/NCCA.2015.12

[7] Vojtech Cima, Bruno Grazioli, SeÃin Murphy, Thomas Michael Bohnert, "Adding energy efficiency to Openstack", 2015 Sustainable Internet and ICT for Sustainability (SustainIT), vol. 00, no. , pp. 1-8, 2015, doi:10.1109/SustainIT.2015.7101358.

[8] D. Thomas Erl, D. Zaigham Mahmood, D. Ricardo Puttini, "Cloud Computing: Concepts, Technology \& Architecture" September 2015.

[9] Yun Zhang, Farhan Patwa, Ravi Sandhu, Bo Tang, "Hierarchical Secure Information and Resource Sharing in OpenStack Community Cloud", 2015 IEEE International Conference on Information Reuse and Integration (IRI), vol. 00, no. , pp. 419-426, 2015, doi:10.1109/IRI.2015.71.

[10] [10] Robayet Nasim, Andreas J. Kassler, "Deploying OpenStack: Virtual Infrastructure or Dedicated Hardware", , vol. 00, no. , pp. 84-89, 2014, doi:10.1109/COMPSACW.2014.18.

[11] David Bernstein, "Cloud Foundry Aims to Become the OpenStack of PaaS", IEEE Transaction on Cloud Computing, vol. 1, no. , pp. 57-60, July 2014, doi:10.1109/MCC.2014.32.

[12] Juan Angel Lorenzo del Castillo, Kate Mallichan, Yahya Al-Hazmi, "OpenStack Federation in Experimentation Multi-cloud Testbeds", , vol. 02, no. , pp. 51-56, 2013, doi:10.1109/CloudCom.2013.103.

[13] Yang Wang, Xiaomin Wang, Jianyong Chen, "On-Demand Security Architecture for Cloud Computing", 
IEEE Computer, vol. 45, no. , pp. 73-78, July 2012, doi:10.1109/MC.2012.120.

[14] V.KRISHNA REDDY1, Dr. L.S.S.REDDY, "Security Architecture of Cloud Computing", International Journal of Engineering Science and Technology (IJEST), ISSN : 0975-5462 Vol. 3 No. 9 September 2011 pp.7149-7155.

[15] A Beloglazov, J Abawajy, R Buyya - Future generation computer systems, Future Generation Computer Systems, Elsevier, Volume 28, Issue 5, May 2012, Pages 755-768.

[16] lan Foster, Rubén S. Montero, Borja Sotomayor, Ignacio M. Llorente, "Virtual Infrastructure Management in Private and Hybrid Clouds", IEEE Internet Computing, vol. 13, no. , pp. 14-22, September/October 2009, doi:10.1109/MIC.2009.119.

[17] Wen-Hwa Liao, Shuo-Chun Su, "A Dynamic VPN Architecture for Private Cloud Computing", 2011 IEEE 4th International Conference on Utility and Cloud Computing (UCC 2011), vol. 00, no. , pp. 409-414, 2011, doi:10.1109/UCC.2011.68.

[18] Sumit Goyal, "Public vs Private vs Hybrid vs Community - Cloud Computing: A Critical Review", I.J. Computer Network and Information Security, 2014, 3, 20-29.

[19] R. Palwe, G. Kulkarni and A. Dongare, -A new approach to hybrid cloud,\|l International Journal of Computer Science and Engineering. 2(1), 1-6, 2012.

[20] L. Cao, X. Liu, M. Liu and K. Han, -Processbased Security Detection Approach for Virtual Machines on Private Cloud Platforms,|| Journal of Networks, 8(6), 1380-1386, 2013.

[21] T.S. Soares, M.A.R. Dantas, D. Macedo and M.A. Bauer, - A data management in a private cloud storage environment utilizing high performance distributed file systems, $\|$ In: Proceedings of the IEEE 22nd International Workshop on Enabling Technologies: Infrastructure for Collaborative Enterprises, 